\title{
Factores asociados a la experiencia de sufrimiento en pacientes con cáncer auanzado
}

\section{Factors flssociated with the Experience of Suffering in Adduanced Cancer Patients Fatores associados à experiência de sofrimento em pacientes com cancro avançado}

\author{
Alicia Krikorian* \\ Universidad Pontificia Bolivariana, Medellín, Colombia \\ Joaquín T. Limonero* \\ Universidad Autónoma de Barcelona, España
}

Doi: dx.doi.org/10.12804/apl33.03.2015.05

\section{Resumen}

Objetivo: Examinar la experiencia de sufrimiento y su relación con problemas físicos, psicológicos, sociales y espirituales en pacientes oncológicos. Método: 98 enfermos de una institución oncológica en cuidados paliativos participaron en el estudio. Se examinó el grado de sufrimiento, la intensidad y amenaza de síntomas y problemas físicos, psicológicos, sociales y espirituales, el grado de ajuste y de conciencia de enfermedad y las estrategias de afrontamiento. Se realizaron análisis estadísticos descriptivos y comparativos. Resultados: Aproximadamente el 50\% de los participantes manifestaron un sufrimiento moderado. Los problemas más frecuentes fueron malestar general, astenia, alteración del estado de ánimo, pérdida de la autonomía, tristeza, preocupación por el futuro de la familia y sufrimiento. Un sufrimiento moderado a severo se asoció con mayor dificultad en el ajuste general y uso de la estrategia de afrontamiento de pensamiento desiderativo. Un menor sufrimiento se asoció con estrategias de reestructuración cognitiva y evitación de problemas. Conclusiones: Se resalta la importancia de atender los aspectos subjetivos de la experiencia. Cualquier síntoma o problema tiene el potencial de generar sufrimiento, en la medida en que sea percibido como amenazante, $y$ los procesos regulatorios con los que cuente la persona impidan la adaptación. Palabras clave: sufrimiento; cáncer; final de vida; malestar emocional; factores asociados; psicológico; social; físico; espiritual.

\section{flbstract}

Objective: To examine the experience of suffering and its relation with physical, psychological, social and spiritual problems in advanced cancer patients. Methods: Ninety-eight patients attending the palliative care unit of an oncologic institution participated in the study. The level of suffering and the intensity and threat perceived from symptoms and a series of physical, psychological, social

* Alicia Krikorian, miembro del Grupo de Dolor y Cuidado Paliativo, Escuela de Ciencias de la Salud, Universidad Pontificia Bolivariana, Medellín, Colombia; Joaquín T. Limonero, miembro del Grupo de Investigación en Estrés y Salud (GIES), Facultad de Psicología, Universidad Autónoma de Barcelona, España.

La correspondencia relacionada con este artículo debe ser enviada a Alicia Krikorian, Escuela de Ciencias de la Salud, Oficina de Posgrados, calle 78B \# 72A-109, Universidad Pontificia Bolivariana, Medellín, Colombia. Correo electrónico: aliciakriko@gmail.com

Cómo citar este artículo: Krikorian, A. \& Limonero, J. T. (2015). Factores asociados a la experiencia de sufrimiento en pacientes con cáncer avanzado. Avances en Psicología Latinoamericana, 33(3), 423-438. doi: dx.doi.org/10.12804/ap133.03.2015.05 
and spiritual problems were examined, as well as adjustment levels, illness awareness and coping strategies. Descriptive and comparative statistics were obtained. Results: Nearly $50 \%$ of participants manifested moderate suffering. The most frequent problems encountered were: general distress, asthenia, emotional distress, loss of autonomy, sadness, concern for the family's future and suffering. Severe suffering was associated to adjustment problems and use of wishful thinking. Lower levels of suffering were associated with cognitive restructuring and avoidance strategies. Conclusions: Results indicate the importance of focusing on the subjective aspects of the illness experience. Any symptom or problem has the potential to become a source of suffering when perceived as threatening and when the person's regulatory processes are insufficient for adaptation.

Keywords: suffering; cancer; end-of-life; distress; associated factors; psychological; social; physical; spiritual.

\section{Resumo}

Objetivo: Examinar a experiência de sofrimento e sua relação com problemas físicos, psicológicos, sociais e espirituais em pacientes oncológicos. Método: 98 doentes de uma instituição oncológica em cuidados paliativos participaram no estudo. Examinou-se o nível de sofrimento, a intensidade e ameaça de sintomas e problemas físicos, psicológicos, sociais e espirituais, o grau de ajuste e de consciência de doença e as estratégias de afrontamento. Realizaram-se análises estatísticos descritivos e comparativos. Resultados: aproximadamente o $50 \%$ dos participantes manifestaram um sofrimento moderado. Os problemas mais frequentes foram mal-estar geral, astenia, alteração do estado de ânimo, perdida da autonomia, tristeza, preocupação pelo futuro da família e sofrimento. Um sofrimento moderado a severo associou-se a maior dificuldade no ajuste geral e uso da estratégia de afrontamento de pensamento desiderativo. Um menor sofrimento associou-se a estratégias de restruturação cognitiva e evitação de problemas. Conclusões: ressalta-se a importância de atender aos aspetos subjetivos da experiência. Qualquer sintoma ou problema tem o potencial de gerar sofrimento na medida em que seja percebido como ameaçante, e os processos regulatórios com os que a pessoa conte impeçam a adaptação.

Palavras-chave: sofrimento; cancro; final de vida; mal-estar emocional; fatores associados; psicológico; social; físico; espiritual.

Las enfermedades que amenazan la vida, como el cáncer, se caracterizan por una serie de síntomas y experiencias que pueden contribuir en diferentes formas al sufrimiento de los enfermos (Stein, Syrjala \& Andrykowski, 2008). El sufrimiento, especialmente al final de la vida, tiende a ser muy prevalente, pues ocurre hasta en un $90 \%$ de los pacientes (Baines \& Norlander, 2000). Aproximadamente el $60 \%$ de los pacientes en el contexto de los cuidados paliativos (CP) experimenta un sufrimiento de moderado a severo (Kuuppelomaki \& Lauri 1998; Benedict, 1989; Wilson et al., 2007).

El alivio del sufrimiento y la mejoría del bienestar son los dos objetivos prioritarios de los CP (World Health Organization, 2002; Woodruff, 2004). Sin embargo, estos conceptos son subjetivos y dinámicos en cada paciente, especialmente al final de la vida, y varían de acuerdo con el contexto sociocultural (Albers et al., 2010). El sufrimiento se ha definido como una amenaza a la integridad de la persona (Cassell, 1999) y, más recientemente, como una experiencia multidimensional y dinámica de estrés severo que ocurre ante eventos de amenaza a la integridad de la persona y donde los procesos regulatorios, que normalmente llevarían a la adaptación, resultan insuficientes y causan agotamiento (Krikorian, Limonero, Román, Vargas \& Palacio, 2014).

Las causas del sufrimiento pueden ser múltiples, dinámicas, sincrónicas y tener un efecto aditivo; los aspectos que generan o contribuyen al sufrimiento son interdependientes aunque puedan diferenciarse, según su naturaleza, en físicos, psicológicos, sociales, culturales y espirituales (Cassell, 1999; Chapman \& Gavrin, 1999; Bayés, Arranz, Barbero, 
\& Barreto, 1996; Woodruff, 2004; Wilson et al., 2007). El sufrimiento emerge de forma integrada, a pesar de que se asocie a múltiples problemas particulares.

Si bien se han estudiado los factores que se asocian con la experiencia de sufrimiento desde cada una de las dimensiones mencionadas (Abraham, 2006; Daneault \& Dion, 2004; Wilson et al., 2007; Krikorian \& Limonero, 2011), son escasos los estudios que realizan una evaluación integrada $y$, menos aún, los desarrollados en Latinoamérica (Silva, 2008) y ninguno, que sepamos, en Colombia.

Dado que los procesos de evaluación e intervención deben tener en cuenta la complejidad, la multidimensionalidad y la subjetividad de los síntomas y experiencias que acompañan el sufrimiento, es esencial conocer profundamente las causas y los factores que contribuyen en un momento determinado a la experiencia de sufrimiento de los pacientes en cada contexto social y cultural, a fin de proporcionar la mejor atención posible. Por ello, el presente estudio tuvo como objetivo evaluar los síntomas físicos y aspectos psicológicos, sociales y espirituales asociados con la experiencia de sufrimiento en un grupo de enfermos con cáncer avanzado/terminal e identificar aquellos que contribuyen al sufrimiento.

\section{Método}

\section{Tipo y diseño de investigación}

Se llevó a cabo un estudio de diseño transversal, descriptivo y correlacional, usando un muestreo por conveniencia.

\section{Participantes}

Los participantes fueron 103 enfermos con cáncer avanzado/terminal (en estadios III o IV y con una expectativa de vida menor a 6 meses, según criterio médico) que fueron atendidos por el equipo de CP en la consulta externa u hospitalaria del Ins- tituto de Cancerología de la Clínica Las Américas (Medellín, Colombia) y participaron en el estudio entre abril y septiembre de 2011. Se llevó a cabo un muestreo por conveniencia. Cinco participantes se descartaron, dado que sus entrevistas fueron interrumpidas por malestar físico. La muestra final estuvo constituida por 98 pacientes.

\section{Instrumentos}

Se recogieron datos sociodemográficos y médicos y se diseñó un instrumento que permitió identificar: el grado de sufrimiento, la intensidad (en una escala de 0 a 10) y el grado de amenaza (en una escala de 0 a 4 ) de los síntomas físicos (dolor, somnolencia, náuseas, astenia, disnea, insomnio, inapetencia y malestar general), psicológicos (estado de ánimo general, ansiedad, miedo, depresión, pérdida del interés, irritabilidad, culpa y pérdida de la autonomía), problemas sociales (sentimientos de soledad/abandono, dificultades de comunicación, sentirse una carga para los demás, satisfacción con el apoyo social percibido, problemas económicos, problemas del entorno social, problemas relacionados con el futuro de la familia) y espirituales (desesperanza, satisfacción con la vida, deseos de morir, sentido de vida, conexión, dignidad y paz consigo mismo). Adicionalmente, se evaluaron ocho estrategias de afrontamiento, el grado de desajuste y conciencia de enfermedad y la ayuda percibida de la entrevista.

Para cuantificar el grado de sufrimiento se empleó la Representación Gráfica de la Enfermedad y Autoevaluación (Büchi, Sensky, Sharpe \& Timberlake, 1998), que es fiable, válida y sensible al cambio terapéutico, y es regularmente usada en el ámbito hospitalario para evaluar sufrimiento (Krikorian, Limonero \& Corey, 2013). Está constituida por un tablero tamaño din-A4 con un disco amarillo de siete centímetros de diámetro en la esquina inferior izquierda. Se le pide al enfermo que imagine que el tablero es su vida actual y que el círculo representa su "sí mismo". Luego se le 
da otro disco de color rojo de cinco centímetros de diámetro que representa su enfermedad, y se le pide que lo ubique en el tablero indicando qué tanto le afecta a sí mismo y su vida actual. La distancia en centímetros que existe entre los centros de los dos círculos, "separación sí mismo-enfermedad", es considerada una medida cuantitativa del sufrimiento: mientras mayor sea la puntuación obtenida, menor será el grado de sufrimiento. A efectos del presente estudio se decidió invertir la puntuación.

Para la evaluación de los aspectos físicos, psicológicos, espirituales y socioculturales se diseñó un instrumento que integra los ítems del cuestionario de Detección del Malestar Emocional (DME) (Maté et al., 2009; Limonero et al., 2012), la Escala de Evaluación Edmonton (ESAS) (Bruera, Kuehn, Miller, Selmser \& MacMillan, 1991; Carvajal, Centeno, Watson \& Bruera, 2011) y la Entrevista Estructurada para la Evaluación de Síntomas y Preocupaciones en Cuidados Paliativos (SISC) (Wilson et al., 2004). Se incluyeron, además, ítems que forman parte de otros instrumentos para evaluar el sufrimiento (Mejía, 1991; Ruijs, Onwuteaka-Philipsen, van der Wal \& Kerkhof, 2009; Schulz et al., 2010). El instrumento final permitió obtener puntuaciones parciales de las subescalas física, psicológica, social y espiritual.

El DME se desarrolló para detectar el malestar emocional en enfermos al final de la vida. Se emplearon las preguntas que evalúan el estado de ánimo y la percepción de afrontamiento de la situación. El DME otorga una puntuación total (0 a 20) formada por la suma de las puntuaciones de las respuestas (Maté et al., 2009). El DME es práctico, de fácil aplicación y presenta una adecuada fiabilidad y valor predictivo, buena sensibilidad y especificidad (Limonero et al., 2012).

El ESAS es un instrumento que evalúa 9 síntomas comunes en cáncer (dolor, fatiga, náuseas, depresión, ansiedad, mareo, apetito, nivel de bienestar y disnea) a través de escalas visuales análogas. Permite obtener una medida global del malestar asociado a los síntomas. Se ha validado en múlti- ples poblaciones y es de frecuente uso en $\mathrm{CP}$ (Bruera et al., 1991; Bruera, Driver \& Elsayem, 2002; Centeno, Noguera, López y Carvajal, 2004). En el presente estudio se usó la versión en español adaptada para diversas culturas (Carvajal et al., 2011).

La SISC (Wilson et al., 2004) es una entrevista que examina síntomas y preocupaciones comunes en pacientes en CP. Este instrumento consta de 13 ítems: dolor, somnolencia, náuseas, debilidad, disnea, pérdida de dignidad, pérdida de control, sentirse una carga para otros, ansiedad, depresión, pérdida del interés, desesperanza y deseos de morir (Wilson et al., 2004), y ha demostrado ser una herramienta válida, confiable y útil en CP (Krikorian et al., 2013).

Se adicionaron los siguientes ítems: apoyo social percibido (retomado del APGAR familiar de Bellon, Delgado, Luna del Castillo \& Lardelli [1996]), insomnio, miedo, irritabilidad, culpa/ remordimiento, sentido de vida y paz consigo mismo (retomados de las escalas de sufrimiento de Schulz et al. [2010]), preocupación por el futuro de la familia y problemas en el barrio de residencia.

Para la evaluación del afrontamiento se utilizó una escala experimental reducida del afrontamiento construida por Limonero (comunicación personal), a partir del Inventario de Estrategias de Afrontamiento (Cano, Rodríguez \& García, 2007). Está conformado por 8 ítems que examinan la frecuencia de uso de una serie de estrategias para afrontar la enfermedad (resolución de problemas, autocrítica, expresión emocional, apoyo social, reestructuración cognitiva, pensamiento desiderativo, retirada social y evitación de problemas) en una escala Likert de 5 puntos.

Se evaluó, asimismo, el grado de conocimiento $y$ conciencia de enfermedad y los pacientes se agruparon en 5 categorías distintas $(0=\sin$ conciencia de enfermedad; 1 = conciencia leve; $2=$ conciencia moderada; 3 = conciencia elevada, y $4=$ conciencia elevada y en paz con la enfermedad). Puntuaciones más bajas indicaban desconocimiento de diagnóstico, pronóstico o gravedad de su situación de salud 
o la intención paliativa de su tratamiento; mientras que puntuaciones más elevadas indicaban un grado de reconocimiento y conciencia del diagnóstico, pronóstico y gravedad de su enfermedad, así como de la cercanía de la muerte. Finalmente, se añadió un ítem que valoraba en una escala de 0 a 10 si la entrevista realizada les había sido de ayuda.

\section{Procedimiento}

Todos los participantes firmaron un consentimiento informado previa explicación del estudio. Se llevó a cabo una entrevista semiestructurada en la que se facilitó la expresión de emociones y necesidades, buscando garantizar la sensibilidad en el trato dada la situación particular de fragilidad de los pacientes. Se pretendió así que la entrevista tuviera un efecto terapéutico en los enfermos y reforzara el proceso del equipo de CP (Gysels, Shipman \& Higginson, 2008). El estudio fue avalado por el Comité de Ética institucional.

\section{Análisis de datos}

Los datos fueron analizados a través del $\mathrm{Pa}$ quete Estadístico para las Ciencias Sociales (SPSS versión 21.0). Se obtuvieron estadísticos descriptivos de las variables sociodemográficas, médicas y clínicas, y se realizaron análisis correlacionales y comparativos.

\section{Resultados}

\section{Participantes}

La mayoría de los enfermos residía en zonas urbanas (87\%). Aproximadamente, la mitad eran de sexo femenino $(57 \%)$ y estaban casados o vivían con su pareja (54\%). En el 97\% de los casos, vivían con la familia y los cuidadores primarios eran los cónyuges, hijos u otros familiares. La edad media de los enfermos fue de 60 años $(D E=$ 14.25; rango 23-87).
Cerca de la mitad de los enfermos tenían estudios primarios. Respecto a su situación laboral, con más frecuencia eran amas de casa, estaban jubilados o pensionados, o trabajaban de forma autónoma. En su gran mayoría indicaron ser católicos practicantes (tabla 1). La mayoría de las entrevistas fueron realizadas en la consulta externa (78.6\%). Los diagnósticos oncológicos más frecuentes fueron: cáncer gastrointestinal, de pulmón, genitourinario y de mama (tabla 2). Se hizo un seguimiento del tiempo de supervivencia desde la entrevista (abril-agosto de 2011) hasta febrero de 2012. Se obtuvieron datos de 61 participantes (62\%): 39 habían fallecido y 22 continuaban en seguimiento. El tiempo promedio aproximado de supervivencia desde el diagnóstico fue 20 meses, y desde la consulta, 3 meses.

\section{Variables de estudio}

La puntuación media de sufrimiento, evaluada a través de la Representación Gráfica de la Enfermedad y Autoevaluación, fue de 12.52 ( $D E=7.42$; rango 0-27). El 26.5\% de los pacientes presentó sufrimiento leve; el $49 \%$, sufrimiento moderado, y el $24.5 \%$, sufrimiento elevado.

Se examinó la intensidad y el grado de amenaza de cada síntoma físico y problema psicológico, social y espiritual. Es de anotar que tanto la intensidad como el grado de amenaza promedio de las variables en todas las dimensiones fueron de moderados a bajos y ambas medidas estuvieron significativamente correlacionadas $(p<.01)$. En la dimensión física, el malestar general, la astenia, la anorexia y el dolor tuvieron las puntuaciones más elevadas tanto en intensidad como en grado de amenaza (figura 1). En la dimensión psicológica, la tristeza, el bajo estado de ánimo y la pérdida de la autonomía fueron los problemas con mayores puntuaciones. Por su parte, en la dimensión social, la preocupación por el futuro de la familia fue el único problema con una intensidad y amenaza medias; mientras que los demás obtuvieron puntuaciones 


\section{flicia Krikorian, Joaquín T. Limonero}

Tabla 1

Datos sociodemográficos: estadísticos descriptivos $(N=98)$

\begin{tabular}{|c|c|c|c|c|c|c|c|c|}
\hline \multirow[b]{2}{*}{ Variable } & \multirow[b]{2}{*}{ Media } & \multirow[b]{2}{*}{ Mediana } & \multirow[b]{2}{*}{$D E$} & \multirow[b]{2}{*}{ Mín. } & \multirow[b]{2}{*}{ Máx. } & \multicolumn{3}{|c|}{ Percentiles } \\
\hline & & & & & & 25 & 50 & 75 \\
\hline Edad & 60.11 & 62.50 & 14.25 & 23 & 87 & 52 & 62 & 69 \\
\hline \# hijos & 3.59 & 3.00 & 3.19 & 0 & 15 & 1 & 3 & 4 \\
\hline Variable & \multicolumn{3}{|c|}{ Categoría } & \multicolumn{3}{|c|}{ Frecuencia $(N)$} & \multicolumn{2}{|c|}{ Porcentaje } \\
\hline \multirow{2}{*}{ Sexo } & \multicolumn{3}{|l|}{ Femenino } & \multicolumn{3}{|c|}{56} & \multicolumn{2}{|c|}{57.1} \\
\hline & \multicolumn{3}{|l|}{ Masculino } & \multicolumn{3}{|c|}{42} & \multicolumn{2}{|c|}{42.9} \\
\hline \multirow{4}{*}{ Estado civil } & \multicolumn{3}{|l|}{ Soltero } & \multicolumn{3}{|c|}{14} & \multicolumn{2}{|c|}{14.3} \\
\hline & \multicolumn{3}{|c|}{ Casado/pareja de hecho } & \multicolumn{3}{|c|}{53} & \multicolumn{2}{|c|}{54.1} \\
\hline & \multicolumn{3}{|c|}{ Divorciado/separado } & \multicolumn{3}{|c|}{17} & \multicolumn{2}{|c|}{17.3} \\
\hline & Viudo & & & & 14 & & & \\
\hline & Cónyuge & & & & 32 & & & \\
\hline & Hijo & & & & 30 & & & \\
\hline Cuidador & Otro famili & & & & 27 & & & \\
\hline primario & Amigo/con & & & & 3 & & & \\
\hline & Cuidador e & pleado & & & 3 & & & \\
\hline & Sin cuidad & & & & 3 & & & \\
\hline Con & Familia & & & & 95 & & & \\
\hline Con quien vive & Solo & & & & 3 & & & \\
\hline Pecidencin & Zona urban & & & & 85 & & & \\
\hline 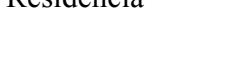 & Zona rural & & & & 13 & & & \\
\hline & Sin estudio & & & & 2 & & & \\
\hline Nivol actudio & Estudios pr & arios & & & 51 & & & \\
\hline tiviver cosiudios & Estudios se & indarios & & & 21 & & & \\
\hline & Estudios su & eriores & & & 24 & & & \\
\hline & Ama de cas & & & & 31 & & & \\
\hline & Jubilado/pe & ionado & & & 27 & & & \\
\hline Siturción lahoral & Autónomo & & & & 24 & & & \\
\hline Siluacton Iavoral & Empleado & & & & 9 & & & \\
\hline & Desemplea & & & & 6 & & & \\
\hline & Otro & & & & 1 & & & \\
\hline & Católico & & & & 90 & & & \\
\hline Afiliación & Cristiano & & & & 4 & & & \\
\hline religiosa & Sin religión & & & & 2 & & & \\
\hline & Testigo de & hová & & & 2 & & & \\
\hline
\end{tabular}




\begin{tabular}{llcc}
\hline \multicolumn{1}{c}{ Variable } & \multicolumn{1}{c}{ Categoría } & Frecuencia $(N)$ & Porcentaje \\
\hline \multirow{2}{*}{$\begin{array}{l}\text { Participación } \\
\text { religiosa }\end{array}$} & Creyente practicante & 86 & 87.8 \\
& Creyente no practicante & 10 & 10.2 \\
& Sin religión & 2 & 2.0 \\
\hline
\end{tabular}

$D E$ : desviación estándar.

Tabla 2

Datos médicos: estadísticos descriptivos $(N=98)$

\begin{tabular}{|c|c|c|c|c|c|c|c|c|}
\hline \multirow[b]{2}{*}{ Variable } & \multirow[b]{2}{*}{ Media } & \multirow[b]{2}{*}{ Mediana } & \multirow[b]{2}{*}{$D E$} & \multirow[b]{2}{*}{ Mín. } & \multirow[b]{2}{*}{ Máx. } & \multicolumn{3}{|c|}{ Percentil } \\
\hline & & & & & & 25 & 50 & 75 \\
\hline $\begin{array}{l}\text { Meses desde el } \\
\text { diagnóstico }\end{array}$ & 30.37 & 15.38 & 40.74 & .27 & 232.23 & 6.10 & 15.38 & 37.13 \\
\hline $\begin{array}{l}\text { Meses desde la } \\
\text { consulta con } \mathrm{CP}\end{array}$ & 8.71 & 4.30 & 12.02 & .00 & 71.27 & .25 & 4.30 & 12.79 \\
\hline $\begin{array}{l}\text { Supervivencia desde } \\
\text { el diagnóstico }\end{array}$ & 19.96 & 13.30 & 18.46 & 1.37 & 73.40 & 7.13 & 13.30 & 28.00 \\
\hline $\begin{array}{l}\text { Supervivencia desde } \\
\text { la entrevista }\end{array}$ & 2.69 & 1.97 & 2.07 & .17 & 8.17 & 1.23 & 1.97 & 3.80 \\
\hline Variable & \multicolumn{4}{|c|}{ Categoría } & & Frecuencia $(N)$ & \multicolumn{2}{|r|}{ Porcentaje } \\
\hline \multirow{3}{*}{ Lugar de la entrevista } & \multicolumn{3}{|c|}{ Consulta externa } & & & 77 & \multicolumn{2}{|r|}{78.6} \\
\hline & \multicolumn{3}{|c|}{ Hospitalización } & & & 21 & \multicolumn{2}{|r|}{21.4} \\
\hline & \multicolumn{3}{|l|}{ Vivos } & & & 22 & \multicolumn{2}{|r|}{22.4} \\
\hline \multirow{8}{*}{$\begin{array}{l}\text { Estatus vital al finalizar } \\
\text { el estudio }\end{array}$} & \multicolumn{3}{|l|}{ Fallecidos } & & & 39 & \multicolumn{2}{|r|}{39.8} \\
\hline & \multicolumn{3}{|l|}{ Sin datos } & & & 37 & \multicolumn{2}{|r|}{37.8} \\
\hline & \multicolumn{3}{|l|}{ Pulmón } & & & 18 & \multicolumn{2}{|r|}{18.4} \\
\hline & \multicolumn{3}{|l|}{ Mama } & & & 14 & \multicolumn{2}{|r|}{14.3} \\
\hline & \multicolumn{3}{|l|}{ Estómago } & & & 9 & \multicolumn{2}{|r|}{9.2} \\
\hline & \multicolumn{3}{|l|}{ Páncreas } & & & 8 & \multicolumn{2}{|r|}{8.2} \\
\hline & \multicolumn{3}{|c|}{ Colon y recto } & & & 13 & \multicolumn{2}{|r|}{13.2} \\
\hline & \multicolumn{3}{|c|}{ Melanoma } & & & 6 & & 6.1 \\
\hline $\begin{array}{l}\text { Diagnóstico } \\
\text { oncológico }\end{array}$ & Ovario & & & & & 5 & & 5.1 \\
\hline & Próstata & & & & & 4 & & 4.1 \\
\hline & Renal & & & & & 4 & & 4.1 \\
\hline & Cuello ute & & & & & 2 & & 2.0 \\
\hline & Esófago & & & & & 2 & & 2.0 \\
\hline & Hematopc & ticos & & & & 4 & & 4.1 \\
\hline & Otros & & & & & 9 & & 9.0 \\
\hline
\end{tabular}


bajas. Finalmente, en la dimensión espiritual, las puntuaciones de los problemas indagados fueron, en general, de baja intensidad y baja amenaza, excepto por el grado de sufrimiento autoinformado y de insatisfacción con la vida, los cuales mostraron una intensidad moderada a baja.

Se examinó el grado de desajuste frente a la enfermedad, obtenido a través del promedio de puntuaciones de los ítems coste percibido, pérdida del control, dificultad en la aceptación de la enfermedad y dificultad en la adaptación (alfa de
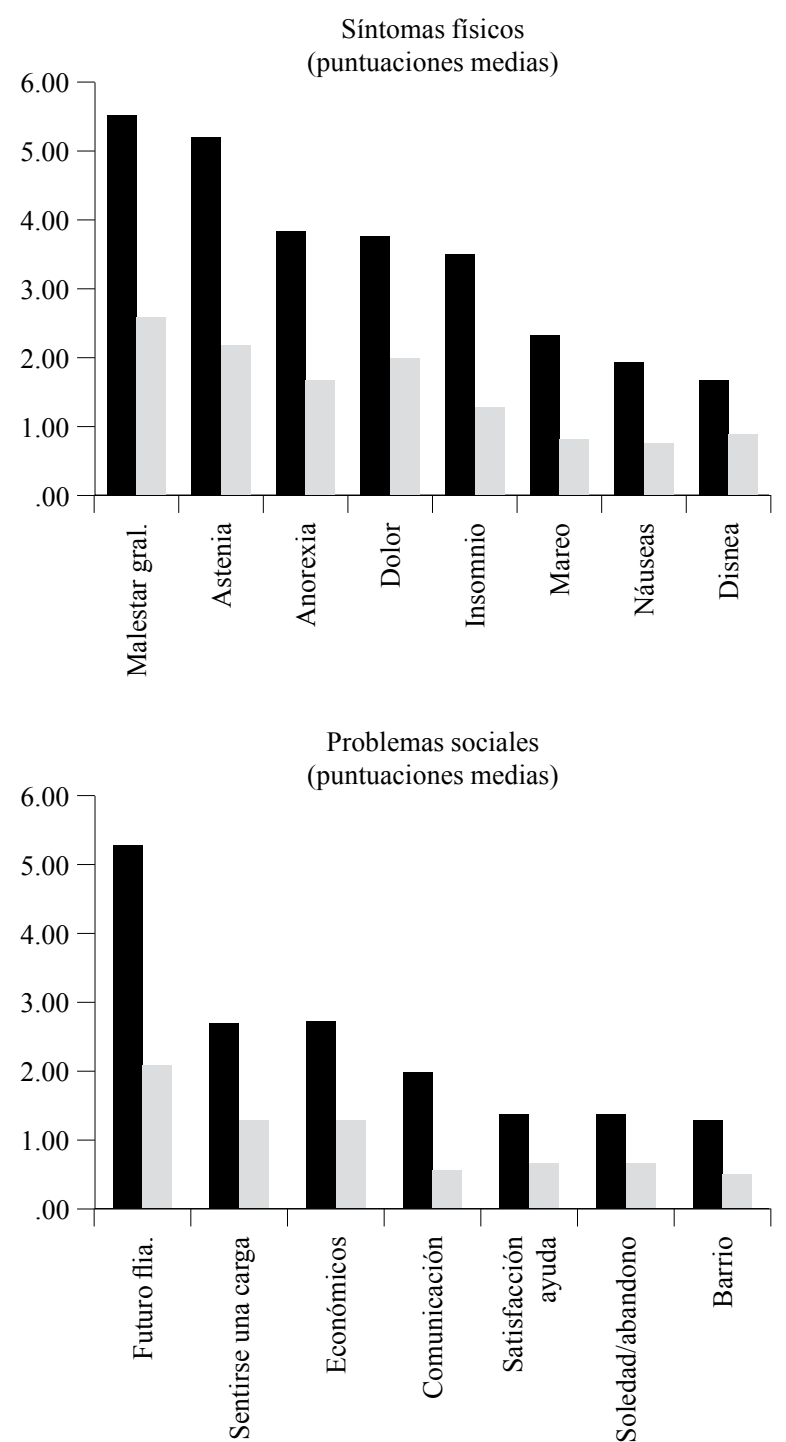

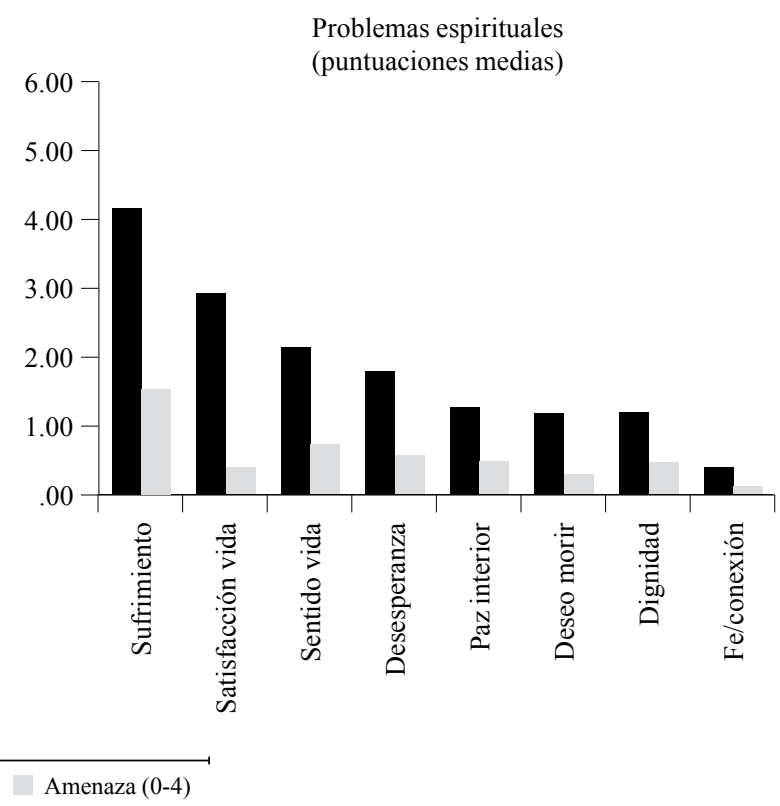

Cronbach $=.82)$. Los resultados indicaron problemas de ajuste de leves a moderados (tabla 3). De los 4 ítems que integraban esta medida, el coste percibido de la enfermedad fue el que obtuvo puntuaciones más elevadas.

En cuanto a las estrategias de afrontamiento, las más frecuentemente utilizadas fueron: esfuerzo por resolver problemas, expresión emocional, ver los aspectos positivos e intentar olvidar. En la tabla 3 se puede observar la media y desviación típica de la frecuencia en el uso de las estrategias evaluadas.

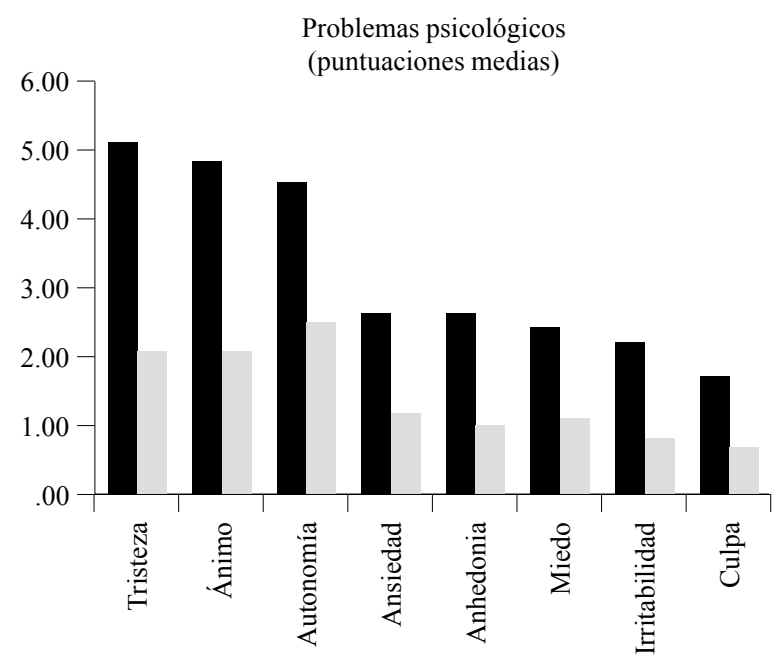

Figura 1. Grado de intensidad y amenaza de los síntomas y problemas en cada dimensión 
Tabla 3. Grado de dificultad en el ajuste a la enfermedad $y$ estrategias de afrontamiento empleadas

\begin{tabular}{|c|c|c|}
\hline Dificultad en el ajuste & $\begin{array}{l}\text { Puntuaciones medias } \\
\quad \text { (rango 0-10) }\end{array}$ & $D E$ \\
\hline Costo percibido & 5.23 & 3.41 \\
\hline Pérdida del control & 4.12 & 3.55 \\
\hline $\begin{array}{l}\text { Dificultad en la } \\
\text { adaptación }\end{array}$ & 4.02 & 3.46 \\
\hline $\begin{array}{l}\text { Dificultad en la } \\
\text { aceptación }\end{array}$ & 3.67 & 3.66 \\
\hline $\begin{array}{l}\text { Dificultad total en el } \\
\text { Ajuste }\end{array}$ & 4.26 & 2.85 \\
\hline $\begin{array}{l}\text { Estrategia de } \\
\text { afrontamiento }\end{array}$ & $\begin{array}{c}\text { Puntuaciones medias } \\
\text { (rango 1-5) }\end{array}$ & $D E$ \\
\hline $\begin{array}{l}\text { Resolución de } \\
\text { problemas }\end{array}$ & 4.12 & 1.06 \\
\hline Expresión emocional & 3.55 & 1.19 \\
\hline $\begin{array}{l}\text { Restructuración } \\
\text { cognitiva }\end{array}$ & 3.50 & 1.37 \\
\hline Evitación de problemas & 3.49 & 1.50 \\
\hline Apoyo social & 2.90 & 1.23 \\
\hline Retirada social & 2.33 & 1.49 \\
\hline $\begin{array}{l}\text { Pensamiento } \\
\text { desiderativo }\end{array}$ & 2.05 & 1.41 \\
\hline Autocrítica & 1.60 & 1.06 \\
\hline
\end{tabular}

Respecto al grado de información y de conciencia de enfermedad, aproximadamente la mitad de los enfermos mostró un grado de información y conciencia de moderado a alto y solo un 7\% manifestó no tener ninguna información o conciencia acerca de su enfermedad. No fue posible obtener esta información en el 15\% del total de los participantes (figura 2).

La mayoría de las personas señalaron que la entrevista les había sido de mucha ayuda (puntuaciones entre 8 y 10). Cabe resaltar que más del $90 \%$ de las personas puntuaron la ayuda percibida con valores superiores a 5 en una escala de 0 a 10 .

\section{Factores asociados al sufrimiento}

Se llevó a cabo un análisis correlacional entre el grado de sufrimiento, medido a través de la Representación Gráfica de la Enfermedad y Autoevaluación, y todas las variables clínicas (tabla 4). Se encontró una correlación positiva y significativa a nivel de .01 con la dimensión física, psicológica, espiritual y la escala total; pero no con la dimensión social o sus componentes. En la dimensión física, el sufrimiento correlacionó de manera directa y significativa con astenia, anorexia y malestar general.

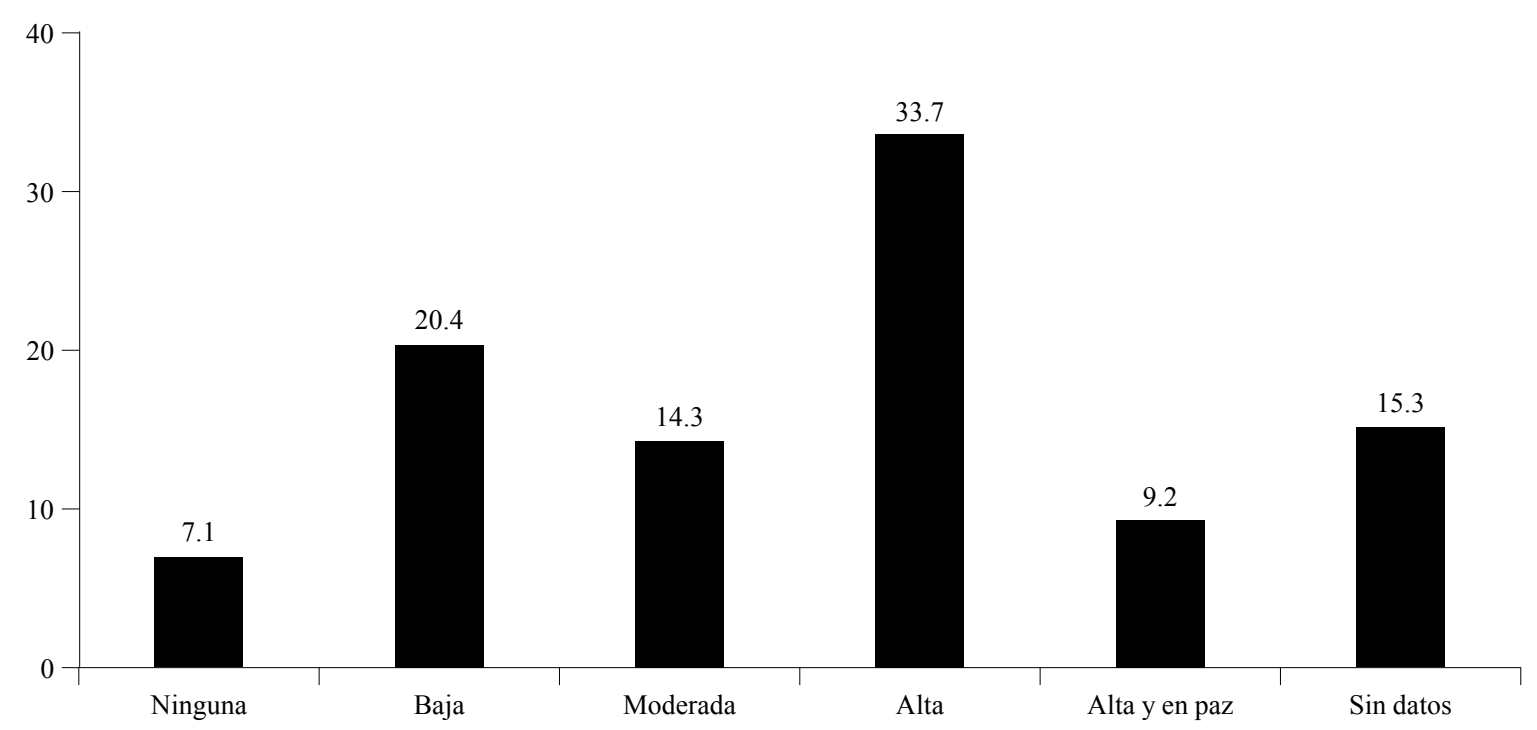

Figura 2. Grado de información y conciencia de enfermedad 
Tabla 4

Comparación entre el grado de sufrimiento y las demás variables clínicas ${ }^{a}$

\begin{tabular}{|c|c|c|c|c|c|}
\hline \multicolumn{2}{|c|}{ Síntomas físicos } & \multicolumn{2}{|c|}{ Problemas psicológicos } & \multicolumn{2}{|c|}{ Problemas sociales } \\
\hline Dolor & .169 & Alteración ánimo & $.332^{* *}$ & Soledad & -.183 \\
\hline Mareo & .066 & Ansiedad & $.347^{* *}$ & P. comunicación & .065 \\
\hline Náuseas & .109 & Miedo & .155 & Carga & .133 \\
\hline Astenia & $.244^{*}$ & Tristeza & $.285^{* *}$ & Insat. ayuda & -.099 \\
\hline Disnea & .109 & Anhedonia & $.209^{*}$ & Futuro & .168 \\
\hline Insomnio & .153 & Irritabilidad & .165 & Probl. económico & .096 \\
\hline Anorexia & $.204^{*}$ & Culpa & $.260^{* *}$ & Probl. barrio & .147 \\
\hline Malestar general & $.199^{*}$ & Pérd. autonomía & $.372^{* *}$ & & \\
\hline \multicolumn{2}{|c|}{ Problemas espirituales } & \multicolumn{2}{|c|}{ Estrategias afrontamiento } & \multicolumn{2}{|l|}{ Otras variables } \\
\hline Pérd. fe & .144 & Res. problemas & -.055 & Dif. adaptación & $.416^{* *}$ \\
\hline Pérd. sentido & $.388^{* *}$ & Exp. emocional & -.086 & Pérdida control & $.488^{* *}$ \\
\hline Pérd. paz & .054 & Apoyo social & .075 & Dif. aceptación & $.459^{* *}$ \\
\hline Insat. vida & .168 & Reestruc. cognitiva & $-.269^{* *}$ & Coste percibido & $.456^{* *}$ \\
\hline Desesperanza & $.389^{* *}$ & Evit. problemas & $-.284^{* *}$ & Total desajuste & $.561^{* *}$ \\
\hline Deseo de morir & $.336^{* *}$ & Autocrítica & $.206^{*}$ & Ayuda entrevista & $-.288^{* *}$ \\
\hline Pérd. dignidad & $.312^{* *}$ & P. desiderativo & $.428^{* *}$ & Conciencia de enfermedad & -.212 \\
\hline Sufrimiento & $.407^{* *}$ & Retirada social & .148 & & \\
\hline \multicolumn{2}{|c|}{ Subescalas } & \multicolumn{2}{|c|}{ Otros instrumentos } & & \\
\hline D. física & $.276^{* *}$ & DME & $.472^{* *}$ & & \\
\hline D. psicológica & $.444^{* *}$ & ESAS & $.292^{* *}$ & & \\
\hline D. social & .093 & SISC total & $.484^{* *}$ & & \\
\hline D. espiritual & $.443^{* *}$ & SISC parcial & $.471^{* *}$ & & \\
\hline Escala total & $.423^{* *}$ & & & & \\
\hline
\end{tabular}

En la dimensión psicológica, la correlación fue directa y significativa con pérdida de la autonomía, ansiedad, alteración del ánimo, tristeza, culpa y anhedonia. En la dimensión espiritual correlacionó directa y significativamente con desesperanza, pérdida de sentido, deseo de morir y pérdida de la dignidad. Asimismo, el grado de sufrimiento mostró una fuerte correlación con todos los ítems que evaluaban las dificultades en el ajuste a la enfermedad, así como la medida compuesta de desajuste. En cuanto a las estrategias de afrontamiento empleadas, se observó una relación significativa y directa entre el sufrimiento y el pensamiento desiderativo y la autocrítica; mientras que la relación fue inversa con evitación de problemas y reestructuración cognitiva. No se encontró relación entre el grado de sufrimiento y el nivel de información y conciencia de enfermedad. Por otra parte, la relación entre el grado de sufrimiento y la percepción de ayuda de la entrevista fue negativa y significativa a nivel de .01, 
lo cual indica que a menor sufrimiento, los participantes percibieron la entrevista de mayor utilidad. Con respecto a las medidas compuestas, se encontró una relación positiva y significativa a nivel de .01 con el DME, las dos versiones del SISC y el ESAS.

\section{Discusión}

La mayoría de los participantes eran de zona urbana, con una educación predominantemente primaria, con disponibilidad de una red de soporte familiar y católicos. La muestra fue representativa de los cánceres más frecuentes de la región y mostró una heterogeneidad, no solo diagnóstica, sino también en cuanto al tiempo desde el diagnóstico, el tiempo que llevaban siendo vistos en CP y la supervivencia (Fornells, McGarrell \& Sala, 2004; Ferlay et al., 2010; Dirección Seccional de Salud de Antioquia, 2012).

El grado de sufrimiento fue moderado en aproximadamente la mitad de los casos. Los niveles de sufrimiento encontrados en otros estudios han sido heterogéneos, en función de las características poblacionales específicas y los instrumentos utilizados. Wilson et al. (2004) y Abraham (2006) reportaron que el grado de sufrimiento señalado por los enfermos fue de bajo a moderado (usando escalas tipo Likert); mientras que los niveles de sufrimiento en los estudios de Benedict (1989), Kuuppelomäki y Lauri (1998) y Wouters, Reimus, van Nunen, Blokhorst \& Vingerhoets (2008), medido a través de la Representación Gráfica de la Enfermedad y Autoevaluación fueron de moderados a severos.

Si bien la intensidad y amenaza de los síntomas y problemas en cada dimensión no fue muy elevada, todos los problemas evaluados fueron experimentados en alguna medida por un número significativo de enfermos, a la vez que hubo una coexistencia de experiencias de malestar. Este hallazgo es común en etapas avanzadas de la enfermedad y usualmente ocurre un efecto aditivo que contribuye al incremento en el sufrimiento
(Abraham, 2006; Wilson et al., 2007; Woodruff, 2004).

Los niveles elevados de sufrimiento se asociaron, al igual que lo encontrado en el estudio de Wilson et al. (2007), con problemas de índole biomédica, psicológica y espiritual: astenia, ansiedad, tristeza, anhedonia, desesperanza, deseo de morir, pérdida de la dignidad, pérdida del control y dificultad en la adaptación a la enfermedad; hecho que demuestra la necesidad de realizar una intervención integral a través de un equipo multidisciplinario.

Si bien los problemas más frecuentemente manifestados por los enfermos en el presente estudio se asemejan a lo encontrado en otras investigaciones (Benedict, 1989; Delgado-Guay, Parsons, Li, Palmer \& Bruera, 2009; Kwon et al., 2006; Kuuppelomaki \& Lauri, 1998; Oi-Ling, Man-Wah \& KamHung, 2005; Portenoy et al., 1994), es importante tener en cuenta que no existen síntomas universalmente amenazantes y que el malestar asociado a cada problema depende del significado atribuido por cada individuo en un momento particular (Bayés \& Limonero, 1999; Limonero, 1994).

El grado de sufrimiento mostró una correlación significativa con las dimensiones física, psicológica y espiritual, pero no con la dimensión social. $\mathrm{Si}$ bien no todas las dimensiones contribuyeron de forma equitativa al sufrimiento, sí hubo una estrecha correlación de las dimensiones entre sí. Este hallazgo corrobora la concepción teórica de que la disrupción en una de las dimensiones genera alteraciones simultáneas en las demás, pues afecta a la persona como un todo (Chochinov, 2006; Woodruff, 2004), y aporta evidencia adicional acerca de la multidimensionalidad de la experiencia de sufrimiento (Krikorian, Limonero \& Maté, 2011).

El hecho de que no se haya encontrado una fuerte relación entre la dimensión social y el grado de sufrimiento no es de extrañar, si se tiene en cuenta que los enfermos contaban con una adecuada disponibilidad de apoyo sociofamiliar, lo que manifiesta la tendencia cultural existente en Colombia a proteger y acompañar activamente a las personas 
enfermas (Vélez, 2008). En este sentido, dicho resultado podría ser un indicador de la disponibilidad y calidad de la red de apoyo social. En consonancia con ello, las personas con cáncer que cuentan con un buen apoyo sociofamiliar experimentan menor malestar y mayor bienestar asociado a la enfermedad (Crothers, Tomter \& Garske, 2005).

Las personas que experimentaron mayor sufrimiento presentaron también mayor dificultad en el ajuste general, en la adaptación y aceptación de la enfermedad, mayor pérdida del control y mayor coste personal percibido. Estos resultados coinciden con lo manifestado por los enfermos en el estudio de Wilson et al. (2007), así como con los hallazgos de otros estudios, donde personas con dificultades para lograr la aceptación y adaptación a su enfermedad y para percibir control en la experiencia presentan mayor malestar emocional y espiritual, y un sufrimiento moderado a severo (Black \& Rubinstein, 2004; Chochinov, Tataryn, Wilson, Ennis \& Lander, 2000; Mystakidou et al., 2010; Ray et al., 2006; Thompson et al., 2009). La dificultad en la adaptación y aceptación de la enfermedad, en conjunto con la pérdida de control y un alto coste percibido, se pueden entender como manifestaciones del agotamiento de los recursos personales para afrontar la enfermedad así como la indefensión producida por la enfermedad. En este caso, la relación hallada entre las dificultades en el ajuste y un mayor sufrimiento estarían en consonancia con las definiciones del sufrimiento de Chapman y Gavrin (1999), Cassell (1999), y con la visión integral del sufrimiento de Krikorian y Limonero (2012).

La estrategia de pensamiento desiderativo se asoció con un mayor sufrimiento; mientras que las estrategias de reestructuración cognitiva y evitación de problemas, con un menor sufrimiento. Este resultado coincide con lo observado en otros estudios (González, Lacasta, Ordóñez \& Belda-Iniesta, 2006; Thompson et al., 2009; O’Brien \& Moorey, 2010), en los cuales aquellos enfermos que se focalizaban en el presente buscaban mantener un buen ánimo y pensar en los aspectos positivos, aceptaban mejor su enfermedad y tenían un menor sufrimiento. Si bien se ha considerado que la evitación de problemas suele ser una estrategia desadaptativa y que se asocia con menor control percibido y menor bienestar (Cano et al., 2007; Folkman \& Greer, 2000), para los enfermos que participaron en este estudio se relacionó con un menor sufrimiento. Por tanto, podría estar sirviendo como mecanismo de protección transitorio en conjunto con la reestructuración cognitiva. Posiblemente, focalizarse en los aspectos positivos de la experiencia, así como evitar pensar o hablar acerca de una enfermedad con la que ya han convivido durante mucho tiempo, les permita mantener una sensación de normalidad en la vida cotidiana y dar continuidad a su existencia, pues se constituyen en estrategias centradas en el significado (Folkman \& Greer, 2000).

Casi la mitad de los participantes presentaron niveles de moderados a altos en el grado de información y conciencia de enfermedad. Sin embargo, no hubo relación entre esta variable y el sufrimiento. Se ha observado que la relación entre la conciencia de enfermedad, el malestar emocional y el sufrimiento suele ser compleja y depende de una serie de factores que interactúan entre sí (Chochinov et al., 2000). Las personas con enfermedad avanzada varían en el grado de información, conciencia y aceptación de su situación. En algunos casos, la conciencia de estar enfermo se suma a dificultades para aceptarlo, lo que genera malestar emocional y espiritual (Mack et al., 2008). En otros casos, la conciencia de enfermedad, junto con una aceptación serena, llevan a mayor bienestar emocional y espiritual (Mack et al., 2008; Thompson et al., 2009). Este hallazgo señala la necesidad de atender el proceso personal de cada individuo con respecto no solo a cómo entiende su enfermedad, sino mejor a cómo la afronta, y si ello le permite adaptarse exitosamente a su situación, sentir bienestar, etc.

Este estudio presenta algunas limitaciones. Primero, dado su diseño transversal, descriptivo y correlacional, fue posible identificar relaciones 
entre las variables, pero no es posible comprobar su causalidad. Segundo, hubo una mayor focalización en aspectos negativos de la experiencia y su contribución al sufrimiento. Futuros estudios deberían examinar el rol protector de aspectos positivos de la experiencia. No se observaron relaciones entre las variables sociales y el sufrimiento, dada la disponibilidad y calidad del apoyo sociofamiliar en los participantes de este estudio. En otras poblaciones con características sociales menos afortunadas, es posible que dicha variable mantenga relación más estrecha con el grado de sufrimiento.

A pesar estas limitaciones, los resultados obtenidos en el presente estudio tienen importantes implicaciones clínicas, dado que resaltan la importancia y necesidad de atender los aspectos subjetivos de la experiencia de cada enfermo. Cualquier síntoma o problema, de origen físico, psicológico, social o espiritual, tiene el potencial de generar sufrimiento en la medida en que sea percibido como amenazante y los procesos regulatorios con los que cuente la persona no le permitan afrontar y adaptarse a su situación. Por tanto, la evaluación e intervención deberán tener siempre en cuenta las diferencias individuales, no solo en cuanto a los factores biomédicos o socioculturales, sino también en cuanto a las variables de personalidad, el significado existencial de la enfermedad, los recursos y estrategias con las que cuente cada enfermo, entre muchas otras variables específicas al momento particular del encuentro. Esto resalta, en particular, la importancia de la participación activa de profesionales en psicología entrenados dentro del equipo asistencial. $\mathrm{Su}$ aporte no solo favorece la oportuna evaluación e intervención sobre el sufrimiento existente, sino que también facilita la prevención del sufrimiento innecesario al abordar y manejar el grado de amenaza percibido y promover un afrontamiento adaptativo a lo largo de la trayectoria de la enfermedad.

Finalmente, la atención de todas las necesidades del enfermo con enfermedad avanzada o al final de la vida requiere la intervención de un equipo multidisciplinar o, en lo posible, interdisciplinar. De igual manera, los miembros del equipo de cuidados paliativos deberán facilitar la implicación de la familia en el cuidado del enfermo, a la vez que atiendan a sus necesidades, puesto que el alivio del sufrimiento de unos potenciará el alivio del sufrimiento de los otros.

\section{Referencias}

Abraham, A. B. (2006). Suffering at the end of life in the setting of low physical symptom distress. Journal of Palliative Medicine, 9(3), 658-665.

Albers, G., Echteld, M. A., de Vet, H. C., Onwuteaka-Philipsen, B.D., van der Linden, M. H., \& Deliens, L. (2010). Evaluation of quality-of-life measures for use in palliative care: a systematic review. Palliative Medicine, 24(1), 17-37.

Baines, B. K., \& Norlander, L. (2000). The relationship of pain and suffering in a hospice population. American Journal of Hospice and Palliative Care, 17, 319-326.

Bayés, R., Arranz, P., Barbero, J., \& Barreto, P. (1996). Propuesta de un modelo integral para una intervención terapéutica paliativa. Medicina Paliativa, 3, 114-121.

Bayés, R., \& Limonero, J. T. (1999). Prioridad en el tratamiento de los síntomas que padecen los enfermos oncológicos en situación terminal. Medicina Paliativa, 6(1), 19-21.

Bellón, J. A., Delgado, A., Luna del Castillo, J. D., \& Lardelli, P. (1996). Validez y fiabilidad del cuestionario de función familiar Apgar-familiar. Atención Primaria, 18(6), 289-296.

Benedict, S. (1989). The suffering associated with lung cancer. Cancer Nursing, 12, 34-40.

Black, H. K., \& Rubinstein, R. L. (2004). Themes of suffering in later life. Journal of Gerontology: Social Sciences, 59B(1), S17-S24.

Bruera, E., Driver, L., \& Elsayem, A. (2002). The M.D. Anderson symptom control and palliative care handbook. Houston: The University of Texas, MD Anderson Cancer Center. 
Bruera, E., Kuehn, N., Miller, M. J., Selmser, P., \& Macmillan, K. (1991). The Edmonton Symptom Assessment System (ESAS): A simple method for the assessment of palliative care patients. Journal of Palliative Care, 7(2), 6-9.

Büchi, S., Sensky, T., Sharpe, L., \& Timberlake, N. (1998). Graphic representation of illness: a novel method of measuring patients' perceptions of the impact of illness. Psychotherapy and Psychosomatics, 67(4-5), 222-225.

Cano, F. J., Rodríguez, L., \& García, J. (2007). Adaptación española del Inventario de Estrategias de Afrontamiento. Actas Españolas de Psiquiatría, 35(1), 29-39.

Carvajal, A., Centeno, C., Watson, R., \& Bruera, E. (2011). A comprehensive study of psychometric properties of the Edmonton Symptom Assessment System (ESAS) in Spanish advanced cancer patients. European Journal of Cancer, 47(12), 1863-1872.

Cassell, E. J. (1999). Diagnosing suffering: a perspective. Annals of Internal Medicine, 131(7), 531-534.

Centeno, C., Noguera, A., López, B., \& Carvajal, A. (2004). Algunos instrumentos de evaluación utilizados en cuidados paliativos (I): el Cuestionario de Evaluación de Síntomas de Edmonton (ESAS). Medicina Paliativa, 1, 239-245.

Chapman, C. R., \& Gavrin, J. (1999). Suffering: The contributions of persistent pain. The Lancet, 353, 233-237.

Chochinov, H. M. (2006). Dying, dignity, and new horizons in palliative end-of-life care. Cancer, $56,84-103$.

Chochinov, H. M., Tataryn, D. J., Wilson, K. G., Ennis, M., \& Lander, S. (2000). Prognostic awareness and the terminally ill. Psychosomatics, 41(6), 500-504.

Crothers, M. K., Tomter, H. D., \& Garske, J. P. (2005). The relationships between satisfaction with social support, affect balance, and hope in cancer patients. Journal of Psychosocial Oncology, 23, 103-118.
Daneault, S., \& Dion, D. (2004). Suffering of gravely ill patients: An important area of intervention for family physicians. Canadian Family Physician, 50, 1343-5, 1348-1350.

Delgado-Guay, M., Parsons, H. A., Li, Z., Palmer, J. L., \& Bruera, E. (2009). Symptom distress in advanced cancer patients with anxiety and depression in the palliative care setting. Supportive Care Cancer, 17, 573-579.

Dirección Seccional de Salud de Antioquia. (2012). Registro poblacional de cáncer de Antioquia: datos preliminares 2007-2009. Recuperado de: htttp://www.dssa.gov.co

Ferlay, J., Shin, H. R., Bray, F., Forman, D., Mathers, C., \& Parkin, D. M. (2010). Globocan (2008) v1.2, Cancer Incidence and Mortality Worldwide: IARC CancerBase No. 10. Lyon, Francia: International Agency for Research on Cancer. Recuperado de: http://globocan.iarc.fr

Folkman, S., \& Greer, S. (2000). Promoting psychological well-being in the face of serious illness: When theory, research and practice inform each other. Psychooncology, 9, 11-19.

Fornells, H., McGarrell, D., \& Sala, R. (2004). Cancer in developing countries. En E. Bruera, L. DeLima, R. Wenk \& W. Farr (Eds.), palliative care in developing countries: Principles and practice (pp. 67-94). Texas: IAHPC Press.

González, M., Lacasta, M. A., Ordóñez, A., \& Belda-Iniesta, C. (2006). Control of oncologic pain in relief of suffering: Our experience. Clinical and Translational Oncology, 8(7), 525-532.

Gysels, M., Shipman, C., \& Higginson, I. J. (2008). Is the qualitative research interview an acceptable medium for research with palliative care patients and carers? BMC Medical Ethics, 9, 7.

Krikorian, A., \& Limonero, J.T. (2012). An integrated view of suffering in palliative care. Journal of Palliative Care, 28(1), 41-49.

Krikorian, A., Limonero, J. T., \& Corey, M. T. (2013). Suffering assessment: A review of available instruments for use in palliative care. Journal 
of Palliative Medicine, 16(2), 130-142. doi. 10.1089/jpm.2012.0370

Krikorian, A., Limonero, J. T., \& Maté, J. (2011). Suffering and distress at the end-of-life. Psychooncology, 21(8), 799-808. doi: 10.1002/pon.2087

Krikorian, A., Limonero, J. T., Román, J.P., Vargas, J. J., \& Palacio, C. (2014). Predictors of suffering in advanced Cancer. American Journal of Hospice and Palliative Care, 31, 534-554.

Kuuppelomaki, M., \& Lauri, S. (1998). Cancer patients' reported experiences of suffering. Cancer Nursing, 21, 364-369.

Kwon, Y. C., Yun, Y. H., Lee, K. H., Son, K. Y., Park, S. M., Chang, Y. J., Cleeland, C. S. (2006). Symptoms in the lives of terminal cancer patients: Which is the most important? Oncology, 71, 69-76.

Limonero, J. T. (1994). Evaluación de aspectos perceptivos y emocionales en la proximidad de la muerte. Tesis doctoral publicada en microfilm. Bellaterra: Universidad Autónoma de Barcelona. Limonero, J. T., Mateo, D., Maté, J., Bayés, R., González-Barboteo, J., Bernaus, M., Viel, S. (2012). Evaluación de las propiedades psicométricas de la escala de Detección de Malestar Emocional-DME en pacientes con enfermedad oncológica avanzada. Gaceta Sanitaria, 26(2), 145152. doi:10.1016/j.gaceta.2011.07.016

Mack, J. W., Nilsson, M., Balboni, T., Friedlander, R. J., Block, S. D., Trice, E., \& Prigerson, H. G. (2008). Peace, Equanimity, and Acceptance in the Cancer Experience (PEACE): Validation of a scale to assess acceptance and struggle with terminal illness. Cancer, 112(11), 2509-2517.

Maté, J., Mateo, D., Bayés, R., Bernaus, M., Casas, C., González-Barboteo, J., Viel. S. (2009). Elaboración y propuesta de un instrumento para la detección de malestar emocional en enfermos al final de la vida. Psicooncología, 6(2-3), 507-518.

Mejía, D. (1991). Salud familiar para América Latina. Bogotá: Asociación Colombiana de Facultades de Medicina.
Mystakidou, K., Tsilika, E., Parpa, E., Gogou, P., Theodorakis, P., \& Vlahos, L. (2010). Self-efficacy beliefs and levels of anxiety in advanced cancer patients. European Journal of Cancer Care, 19, 205-211.

O'Brien, C. W., \& Moorey, S. (2010). Outlook and adaptation in advanced cancer: a systematic review. Psychooncology, 19(12), 1239-1249. doi: 10.1002/pon.1704

Oi-Ling, K., Man-Wah, D., \& Kam-Hung, D. (2005). Symptom distress as rated by advanced cancer patients, caregivers and physicians in the last week of life. Palliative Medicine, 19, 228-233.

Portenoy, R. K., Thaler, H. T., Kornblith, A. B., Lepore, J. M., Friedlander-Klar, H., Coyle, Scher, H. (1994). Symptom prevalence, characteristics and distress in a cancer population. Quality of Life Research, 3, 183-189.

Ray, A., Block, S. D., Friedlander, R. J., Zhang, B., Maciejewski, P. K., \& Prigerson, H.G. (2006). Peaceful awareness in patients with advanced cancer. Journal of Palliative Medicine, 9(6), 1359-1368.

Ruijs, K. D., Onwuteaka-Philipsen, B. D., van der Wal, G., \& Kerkhof, A. J. (2009). Unbearability of suffering at the end of life: the development of a new measuring device, the SOS-V. BMC Palliative Care, 3(8), 16-26.

Schulz, R., Monin, J. K., Czaja, S. J., Lingler, J. H., Beach, S. R., Martire, L. M., Cook, T. B. (2010). Measuring the experience and perception of suffering. Gerontologist, 50(6), 774-784.

Silva, L. C. da (2008). Câncer de mama e sofrimento psicológico: aspectos relacionados ao feminino. Psicologia em Estudo, 13(2), 231-237.

Stein, K. D., Syrjala, K. L., \& Andrykowski, M. A. (2008). Physical and psychological long-term and late effects of cancer. Cancer, 112, 25772592.

Thompson, G. N., Chochinov, H. M., Wilson, K. G., McPherson, C. J., Chary, S., O'Shea, F. M.,Macmillan, K. A. (2009). Prognostic acceptance and the well-being of patients receiving palliative 
care for cancer. Journal of Clinical Oncology, 27(34), 5757-5762.

Vélez, M. C. (2008). Caracterización de los cuidadores. En Grupo de Dolor y Cuidado Paliativo UPB, Dolor y sufrimiento: perspectivas desde los cuidados paliativos (pp. 43-53). Medellín: UPB.

Wilson, K. G., Chochinov, H. M., McPherson, C. J., LeMay, K., Allard, P., Chary, S., Fainsinger, R. L. (2007). Suffering with advanced cancer. Journal of Clinical Oncology, 25(13), 16911697.

Wilson, K. G., Graham, I. D., Viola, R. A., Chater, S., de Faye, B.J., Weaver, L. A., \& Lachance, J. A. (2004). Structured interview assessment of symptoms and concerns in palliative care. Canadian Journal of Psychiatry, 49(6), 350-358.

Woodruff, R. (2004). Palliative care: basic principles. En E. Bruera, L. DeLima, R. Wenk, W. Farr. (Eds.), Palliative care in developing countries: Principles and practice. Texas: IAHPC Press. World Health Organization. (2002). Cancer control program: policies and managerial guidelines (2nd ed.). Geneva: WHO.

Wouters, E. J., Reimus, J. L., van Nunen, A. M., Blokhorst, M. G., Vingerhoets, A. J. (2008). Suffering quantified?: Feasibility and psychometric characteristics of 2 revised versions of the Pictorial Representation of Illness and Self Measure (PRISM). Behavioral Medicine, 34(2), 65-78. 